\title{
Hypocholesterolaemic and hepatoprotective effects of virgin avocado oil in diet-induced hypercholesterolaemia rats
}

\begin{abstract}
ABSTRCT
Hypercholesterolaemia is a chronic disease associated with the development of nonalcoholic fatty liver disease and atherosclerosis. This study aimed to investigate the hypocholesterolaemic and hepatoprotective effects of virgin avocado oil (VAO) using diet-induced hypercholesterolaemia rats. Male Sprague Dawley rats were fed high-cholesterol diet for 4 weeks to induce hypercholesterolaemia. After confirming the establishment of hypercholesterolaemia model, the VAO (450 and $900 \mathrm{mg} \mathrm{kg}-1$ body weight per day) and simvastatin (10 mg kg-1 body weight per day) were given orally while maintaining the high-cholesterol diet for another 4 weeks. Changes in anthropometric, lipid profile, liver biomarkers and liver histology were determined. At the end of the experiment, the serum low-density lipoprotein cholesterol (LDL-C) and triglyceride (TG) levels were significantly reduced, while the high-density lipoprotein cholesterol (HDL-C) level was significantly increased in high-dose VAO- (900 mg kg-1 body weight per day) and simvastatin-treated rats when compared with their respective baseline values. The liver damage index was also markedly reduced in all treated rats. Current findings demonstrated the potential hypocholesterolaemic and hepatoprotective benefits of VAO in the preclinical study. The potential of virgin avocado oil in the treatment and management of hypercholesterolemia.
\end{abstract}

Keyword: Fatty liver disease; Hypocholesterolaemic; Statin; Virgin avocado oil 